\title{
Accessible Five Compartment Body Composition via 3-Dimensional Imaging and Bioelectrical Impedance
}

Jonathan P. BENNETT *, Devon CATALDI, Brandon K QUON, Yong En LIU, Nisa N KELLY, Tom KELLY, Steven B HEYMSFIELD, Andrea K GARBER, Ethan J WEISS, John A SHEPHERD

University of Hawaii Cancer Center, Honolulu HI, USA

https://doi.org/10.15221/21.39

Keywords: Multicompartment body composition, optical body scanning

\section{Background}

Five-compartment modeling of body composition (bone mineral, fat, water, protein, extraosseous mineral masses) is considered to be the criterion in vivo model for body composition assessment. Use of multiple techniques to assess body composition minimizes assumptions (uniform density of fat and fat free mass, hydration of fat free mass, etc.) known to vary based on age, sex, race, and physical activity and allows for a more accurate estimation of body composition in populations where hydration, bone density, or musculature may vary significantly. However, the 5-compartment (5C) model requires multiple technologies including dual-energy X-ray absorptiometry (DXA) for bone mass, airdisplacement plethysmography (ADP) for body volume, deuterium dilution (D2O) for total body water measurement, extraosseous mineral estimation and scale weight. The combination is time-consuming, taking 4 hours for the acquisition alone, and costly, limiting its broad use as a feedback tool for coaches and trainers. To date, simplified multicompartment models of body composition have been explored but poorly validated. The purpose of this study was to compare the standard $5 \mathrm{C}$ model to an accessible version using 3-dimensional optical (3DO) imaging, bioelectrical impedance analysis (BIA) and scale weight, a model named 3DO-5C.

\section{Methods}

Student athletes enrolled in the Da Kine Study at the University of Hawaii, Manoa participated. The target was to recruit 80 athletes ( 40 female) from a variety of collegiate sports where height, weight, musculature, and types of activity/training varied. Each measure for the criterion 5-compartment model was performed including scale weight for body mass $(B M)$, deuterium dilution $\left(D_{2} O\right)$ for total body water (TBW) and soft tissue mineral (Ms) estimation, DXA for bone mineral mass (Mo), and ADP for body volume (BV). Wang's form of the 5-compartment model was used to estimate body fat mass (FM) as $\mathrm{FM}=2.748 * B V-0.715 * T B W+1.129 * M o+1.222 * M s-2.051 * B M(1)$. The simplified 3DO-5C multicompartment model utilizes bioelectrical impedance (BIA), 3-dimensional optical (3DO) scans, and scale weight. BIA was calibrated to TBW and 3-dimensional optical was calibrated to BV. Demographics were modeled to estimate Mo using data from the Shape Up! Adults study dataset. After calibrating the simplified methods, the simplified multicompartment model (3DO-5C) was validated to the criterion Wang-5C.

\section{Results}

Analysis included 68 participants (33 female; mean age $23.3 \pm 4.9$ years) with all measures available. $3 D O$ estimates of body volume was highly accurate to $A D P(R 2=0.99, A D P=1.00 * 3 D O+1.75)$. BIA estimated TBW was also highly associated to deuterium dilution ( $R 2=0.98, \mathrm{D} 2 \mathrm{O}=0.99$ * $\mathrm{BIA}-0.65)$. DXA Mo was estimated using height, weight, and gender $(R 2=0.78)$. With the above substitutions, 3DO$5 \mathrm{C}$ model was highly associated with the criterion model $(\mathrm{r} 2=0.84, \mathrm{RMSE}=2.7 \mathrm{~kg}$ ). There was no significant bias and the results of the model were consistent at all levels of body adiposity observed in this study.

\footnotetext{
* Contact: jb99@hawaii.edu
} 


\section{Discussion and Conclusion}

3DO-5C provides an accessible, cost-effective and accurate measure of 5-compartment body composition. Each measurement was completed in under 2 minutes, meaning the 3DO-5C model was obtained in less than 10 minutes, a significant reduction in comparison to the criterion Wang-5C. This method eliminates a significant portion of error associated with using field methods of body composition assessment. It also eliminates the use of DXA, which may be inaccessible in a variety of settings. Given that bone is predicted in this model and because bone is the most stable component of overall body weight, this method also reduces the error related to bone variation when properly applied across different populations. We aim to continue to build upon this preliminary model in an effort to reduce the error compared to the criterion model. Further work should explore the accuracy of this model in tracking body composition over time. When a criterion $5 \mathrm{C}$ method is available, this simplified 3DO-5C model may be applied for additional follow up testing to reduce the cost and burden associated with the criterion method. This novel multicompartment model affords the ability to provide prompt feedback regarding diet and exercise recommendations and continued research will support its application in a variety of settings including general health, athletics, and medical populations.

\section{References}

1. Wang Z, Pi-Sunyer FX, Kotler DP, Wielopolski L, Withers RT, Pierson RN, Heymsfield SB. Multicomponent methods: evaluation of new and traditional soft tissue mineral models by in vivo neutron activation analysis. The American Journal of Clinical Nutrition. 2002;76(5):968-74. doi: 10.1093/ajcn/76.5.968. 